\title{
A PLURALIDADE EM ENCONTRO ACADÊMICO
}

\author{
Paulo Henrique Maia Machado ${ }^{1}$ \\ Henrique Almeida Santos ${ }^{2}$ \\ Carina Merkle Lingnau ${ }^{3}$
}

\begin{abstract}
RESUMO
O movimento de internacionalização tem deixado os alunos do curso de Licenciatura em Informática da Universidade Tecnológica Federal do Paraná, Francisco Beltrão (UTFPR-FB) bastante curiosos em torno do assunto. Por esta razão, na disciplina de Comunicação Linguística, a tarefa de apresentar algo para a turma, de modo a praticar a oralidade, tornou-se um desafio coletivo e teve como resultado a organização de um encontro sobre o tema em que professores, técnicos administrativos, acadêmicos egressos da UTFPR-FB, além de estudantes da Unioeste/México e um acadêmico colombiano auxiliaram no compartilhamento de experiências em relação à discussão sobre a internacionalização. O objetivo desse artigo é apontar o encontro como forma coletiva de apresentar a internacionalização na universidade. Como metodologia utilizamos pesquisa bibliográfica e documental, além das discussões teóricas sobre globalização em Bauman (1999), internacionalização em Morosini (2006) e Nez e Morosini (2020), além das questões sobre a integração da América Latina articuladas por Perrota (2018) e a aplicação do conceito de soft power utilizado por Nye (2017). Para os resultados e discussões, encontramos em ações como essa uma oportunidade de incorporar os países como o Catar, México e Colômbia como possibilidade de local de produção de conhecimento, ou seja, de internacionalização acadêmica no discurso de internacionalização da academia, que, diferentemente de Portugal, França e Reino Unido, já fazem parte da prática secular de intercâmbio. Ademais, a noção de soft power fortalece ações como esse encontro de modo que se crie um movimento de inclusão para a internacionalização entre países considerados à margem dos tradicionais centros da produção do saber.
\end{abstract}

PALAVRAS-CHAVE: globalização. internacionalização acadêmica. países latinos. soft power.

\section{RESUMEN}

El movimiento de internacionalización ha dejado a los estudiantes del curso de Licenciatura en Ciencias de la Computación en la Universidad Tecnológica Federal de Paraná, Francisco Beltrão (UTFPR-FB) muy curiosos sobre el tema. Por esta razón, en la disciplina de la Comunicación Lingüística, la tarea de presentar algo a la clase, para practicar el habla, se convirtió en un desafío colectivo y resultó en la organización de una reunión sobre el tema en la que los maestros, técnicos administrativos, académicos graduados de UTFPR-FB, además de estudiantes de Unioeste/México y un académico colombiano ayudaron a compartir experiencias sobre la discusión sobre internacionalización. El propósito de este artículo es señalar la reunión como una forma colectiva de presentar la internacionalización en la universidad. Como metodología, utilizamos la investigación bibliográfica y documental, además de las discusiones teóricas sobre la globalización en Bauman (1999), la internacionalización en Morosini (2006) y Nez y Morosini (2020), además de las preguntas sobre la integración de América Latina articuladas por Perrota (2018) y la aplicación del concepto de soft power utilizado por Nye (2017). Para los resultados encontramos en acciones como esta, una oportunidad para incorporar a países como Qatar, México y Colombia como una posibilidad de un lugar de producción de conocimiento, es decir, de internacionalización académica en el discurso de internacionalización de la academia, que, a diferencia de Portugal , Francia y el Reino Unido ya forman parte de la práctica del intercambio secular. Además, la noción de soft power fortalece acciones como esta reunión para que se cree un movimiento de inclusión para la internacionalización entre países considerados fuera de los centros tradicionales de producción de conocimiento.

PALABRAS CLAVES: globalización. internacionalización académica. países latinos. soft power.

\footnotetext{
${ }^{1}$ Graduado em Agronomia pela Universidade Tecnológica Federal do Paraná, campus Dois Vizinhos (UTFPRDV). Graduando em Licenciatura em Informática na UTFPR, campus Francisco Beltrão. Francisco Beltrão, Paraná, Brasil; phmm.paulo@ gmail.com.

${ }^{2}$ Acadêmico da Universidade Tecnológica Federal do Paraná, campus Francisco Beltrão (UTFPR-FB); Francisco Beltrão; Paraná; Brasil; henrique.life.almeida@ hotmail.com.

${ }^{3}$ Doutora em Letras (UEM); Professora de magistério superior na Universidade Tecnológica Federal do Paraná, campus Francisco Beltrão (UTFPR-FB); Francisco Beltrão; Paraná; Brasil; carinadebeltrao @ gmail.com.
} 


\begin{abstract}
The internationalization movement has left the students of the Computer Science undergraduate course at the Federal Technological University of Paraná, Francisco Beltrão (UTFPR-FB) very curious about the subject. For this reason, in the discipline of Linguistic Communication, the task of presenting something to the class, in order to practice speaking, became a collective challenge and resulted in the organization of a meeting on the subject in which teachers, administrative technicians, academics graduating from UTFPR-FB, as well as students from Unioeste/Mexico and a Colombian academic helped to share experiences regarding the discussion on internationalization. The purpose of this article is to point out the meeting as a collective way of presenting internationalization at the university. As methodology, we use bibliographic and documentary research, in addition to theoretical discussions on globalization in Bauman (1999), internationalization in Morosini (2006) and Nez and Morosini (2020), as well as the issues on the integration of Latin America written by Perrota (2018) and the application of the soft power concept used by Nye (2017). For the results and discussions we find in actions like this an opportunity to incorporate countries like Qatar, Mexico and Colombia as a possibility of a locus for knowledge production, that is, of academic internationalization in academia's internationalization discourse, which, differently from Portugal, France and the United Kingdom are already part of the secular exchange practice. Furthermore, the notion of soft power strengthens actions such as this meeting so that an inclusion movement is created for internationalization among countries considered outside the traditional centers of knowledge production.
\end{abstract}

KEYWORDS: globalization. academic internationalization. Latin countries. soft power.

\title{
Introdução
}

A internacionalização é um assunto cada vez mais presente nas universidades, no mercado de trabalho, no mundo globalizado (BAUMAN, 1999). Por esta urgência de internacionalizar que surge em cada edital, em cada notícia, em cada apoio financeiro, a curiosidade e o interesse dos/as acadêmicos/as e assim criam a necessidade de discutirem sobre o assunto.

Desse modo, surgiu na disciplina de Comunicação Linguística da Universidade Tecnológica Federal do Paraná, campus Francisco Beltrão (UTFPR-FB) a vontade de conversar sobre a internacionalização no curso de Licenciatura em Informática, turma 2018.2, curso noturno que tem grande concentração de acadêmicos/as trabalhadores/as e que também querem participar das atividades de mobilidade internacional e das palestras e dinâmicas em relação a essa temática, porém muitas atividades são oferecidas no turno diurno, o que motivou o grupo do curso de Licenciatura a coordenar um encontro em que este assunto fosse mais esclarecido.

A partir dessas conversas lançou-se a semente da internacionalização como algo além da experiência de mobilidade. Para tanto, organizou-se este artigo em: metodologia, localização geográfica, UTFPR-FB e sua internacionalização, o encontro acadêmico, resultados, conclusões e referências. 


\section{Metodologia}

Este estudo tem caráter qualitativo (BAUER \& GASKEL, 2002), estruturado na pesquisa bibliográfica e pesquisa documental. No processo de sistematizar essa investigação fez-se levantamento bibliográfico e pesquisou-se nos documentos disponíveis sobre o encontro (vídeo documentado na plataforma youtube), além de materiais elaborados pela coordenação do encontro. A partir da reunião dos elementos que compuseram a investigação buscou-se analisar os resultados através de conceitos como internacionalização (MOROSINI, 2006; NEZ e MOROSINI, 2020), soft power (NYE, 2017) e integração da América Latina (PERROTA, 2018).

\section{Localização geográfica}

A inserção de mapa em um texto dentro de um momento histórico em que facilmente se pode solucionar dúvidas em plataformas como google.com parece ingênuo, porém destacam-se aqui a intencionalidade do mapa, da imagem, da localização, para reforçar a cartografia de espaços que nem sempre estão fixados na memória eurocêntrica dos/as pesquisadores/as.

Assim, dentre os países citados na experiência de mobilidade internacional presentes no encontro promovido pelos alunos/as do curso de Licenciatura estão o Catar, a Colômbia e o México.

Percebe-se no mapa disponibilizado no trabalho que desses três países mencionados, Catar está geograficamente mais distante, tomando o Brasil como referência, enquanto a Colômbia e o México se encontram mais próximos tanto linguística como culturalmente. 


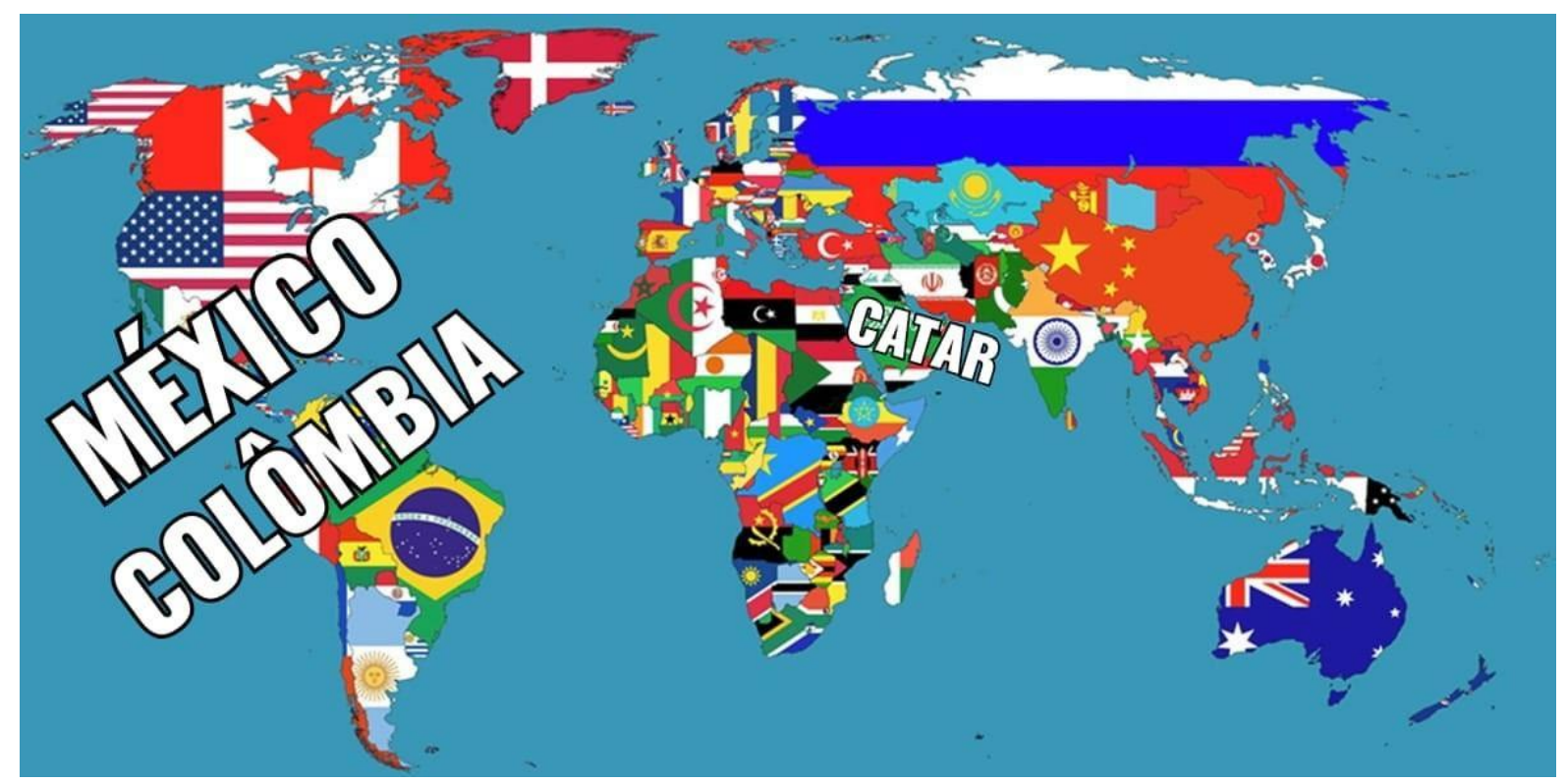

Figura 1: Mapa mundi.

Fonte: https://www.elo7.com.br/quadro-poster-mapa-mundi-bandeiras/dp/E5536A

De acordo com dados retirados do Instituto Brasileiro de Geografia e Estatística (IBGE) o Catar está localizado no Oriente Médio, com uma extensão territorial de 11.586Km2, conta com 2.832.071 habitantes e utiliza o idioma árabe. Esse foi o país de mobilidade do professor de magistério superior da UTFPR-FB durante seu período de formação acadêmica stricto sensu realizados na Universidade Estadual de Maringá (UEM).

Já o México, segundo o IBGE, encontra-se na América Central, tem extensão territorial de $1.964 .375 \mathrm{~km}^{2}$, apresenta 127.575 .529 habitantes e utiliza o idioma espanhol. Esse país recebeu a acadêmica da Universidade Estadual do Oeste do Paraná (UNIOESTE) para seus estudos na área da Geografia.

A Colômbia se destaca no mapa por estar na América do Sul, fazendo divisa com vários países, incluindo o Brasil. De acordo com dados retirados do Instituto Brasileiro de Geografia e Estatística (IBGE) a Colômbia tem uma extensão territorial de $1.138 .910 \mathrm{~km}^{2}$, uma população total de 50.339.443 e o idioma utilizado é o espanhol. Nesta experiência de mobilidade internacional que mencionamos no trabalho, o acadêmico saiu da Colômbia e veio para instituição brasileira cursar o mestrado, o que valoriza a relação de possibilidade do Brasil ser tomado como espaço de mobilidade acadêmica internacional.

Revelando o contexto geográfico do que se quer examinar, passamos à apresentação da UTFPR-FB. 


\section{A UTFPR-FB}

A UTFPR é uma instituição de ensino centenária, mas a entidade enquanto universidade iniciou a partir de 2005 (BRASIL, 2005).

A UTFPR-FB teve início como universidade a partir de 2008, localizada na zona rural do município de Francisco Beltrão oferece cursos de graduação e pós-graduação. Atualmente pode-se encontrar os seguintes cursos de graduação no campus: Licenciatura em Informática (noturno), Engenharia Ambiental (diurno), Engenharia de Alimentos (diurno), Engenharia Química (diurno) e um curso novo ainda não disponível: Agronomia (em implantação).

Em relação aos cursos de pós-graduação, no momento existem os seguintes cursos lato sensu (especialização): Engenharia de Produção, Métodos Matemáticos Aplicados e Inteligência Computacional. Os cursos stricto sensu (mestrado) proporcionados no campus no presente momento são: Programa de Pós-Graduação em Engenharia Ambiental: Análise e Tecnologia Ambiental e Programa de Pós-Graduação em Tecnologia de Alimentos.

Por conta do plano de desenvolvimento institucional (PDI) 2018-2022 (UTFPR) toda a estrutura da universidade é convidada a participar do movimento de internacionalização.

\section{A internacionalização na UTFPR-FB}

Para Morosini (2006, p.192) a internacionalização é "considerada como qualquer esforço sistemático que tenha como objetivo tornar a Educação Superior mais respondente às exigências e desafios relacionados à globalização da sociedade, da economia e do mercado de trabalho". Então não podemos perder de vista a questão econômica que envolve o processo de internacionalização nas instituições de ensino superior (IES), para que o olhar ingênuo das trocas culturais e de conhecimento não sejam os únicos elementos motivadores dessa ação.

A partir do Programa Ciência sem Fronteiras (CsF) criado no governo Dilma Rousseff o movimento de internacionalização tomou forma concreta na UTFPR-FB (LINGNAU e NAVARRO, 2018). No período entre 2012 e 2015 graduandos do campus Francisco Beltrão foram direcionados à Europa e América do Norte.

Após o término do programa outros convênios foram estabelecidos entre a UTFPR e IES internacionais, porém em sua maioria, os convênios foram direcionados para o continente europeu e/ou norte-americano.

Nesse contexto, no momento em que convênios iam tomando forma de colegas indo para o exterior os graduandos do curso de Licenciatura em Informática de 2018.2 ficaram 
interessados e procuraram entender como poderiam partilhar da internacionalização enquanto experiência acadêmica para crescimento e trocas.

\section{O encontro sobre experiências internacionais}

O momento de reunir graduandos do curso de Licenciatura em Informática em um encontro que discutisse algumas possibilidades de mobilidade estudantil internacional (MEI) foi uma oportunidade de pensar a internacionalização Brasil-mundo, assim como mundoBrasil.

Porém a internacionalização não é uma tarefa simples, Alfano (2016, p.1) afirma o que Bauman socializa sobre a influência do mercado capitalista na educação: “[...] o sistema universitário de hoje foi incorporado pela economia de mercado capitalista. Ele serve como um outro mecanismo na reprodução de privilégios e aprofundamento das desigualdades sociais".

Essas relações econômicas também geram efeitos na educação superior e nas ofertas/escolhas realizadas pelas instituições acadêmicas e/ou seus acadêmicos/as, servidores/as, gestores/as, além do mercado de trabalho que acolhe/cria expectativas/necessidades aos trabalhadores/as da produção de conhecimento.

Afinal, como pontuam Nez e Morosini (2020) “a Educação Superior é central para o desenvolvimento social e econômico, é uma condição sine qua non para o desenvolvimento". Assim, considerando que se estabelecem relações de pertencimento ao redor do globo, vivemos em tempos de mercado internacional, o que significa estar conectado e pronto/a para as relações globalizadas. Para Bauman (1999, p.7), a globalização é "um processo irreversível; é também um processo que afeta a todos na mesma medida e da mesma maneira. Estamos todos sendo "globalizados" - e isso significa basicamente o mesmo para todos".

Mas será que esse "mesmo para todos" é o que realmente é melhor para todos/as? Pensando nisso, busca-se no encontro sobre mobilidade internacional trazer convidados/as com experiências heterogêneas em relação ao "estar globalizado", o que significa que não apenas a Europa e a América do Norte estavam presentes, mas América Latina e Ásia.

Nesse quesito, o cientista político Nye (2017, p.2) cunha o conceito soft power (poder brando) como "the ability to affect others by attraction and persuasion rather than just coercion and payment" (tradução livre dos autores: "A capacidade de afetar os outros por atração e persuasão, em vez de somente coerção e pagamento”. A apresentação de países da 
América Latina e da Ásia como possibilidades de mobilidade internacional agrega valor simbólico ao tema até então desconhecido pelo grupo de alunos/as.

Visualiza-se na imagem a seguir, o docente cuja mobilidade internacional para stricto sensu foi o Catar, mostra a imagem de árabes conversando ao fundo, com vestes diferentes das ocidentais, assim como observa-se uma bandeira diferente disposta à frente do professor, é a bandeira do Catar, imagem incomum às campanhas de mobilidade internacional.

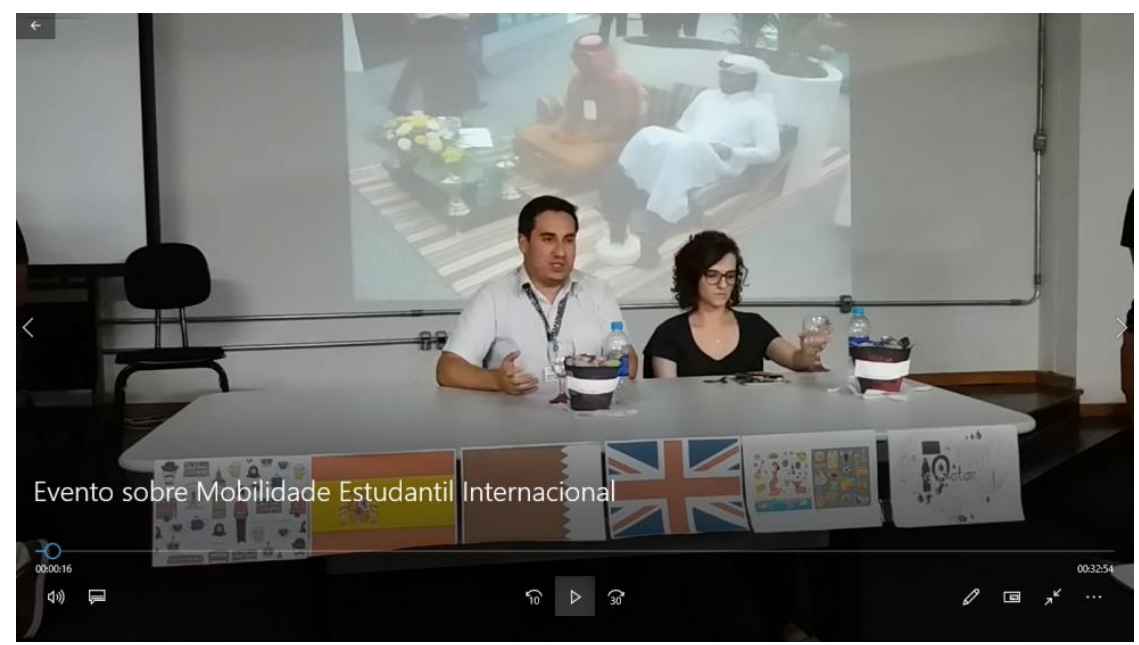

Figura 2 - Catar.

Fonte: https://www.youtube.com/watch?v=YxBZ5jJ9EAM\&t=6s

Ao trazer as questões culturais para seu depoimento/compartilhamento de experiência instaura o que Hoogwaerts (2016, p. 320) pontua sobre a questão da cultura e sua influência nas relações entre países. Ela considera que “institutions' shifting position in society - both online and in their physical manifestations - and their role in the development of soft power, should not be underplayed" (tradução livre dos autores: "A mudança da posição das instituições na sociedade - tanto online quanto em suas manifestações físicas - e seu papel no desenvolvimento do soft power não devem ser subestimados”). Assim, micro-ações como um encontro aparentemente inofensivo, pode causar impactos sociais nas escolhas futuras nos/as envolvidos/as no processo de conhecimento daquilo que não está evidente para o momento intelectual/cultura dos/as participantes do encontro realizado.

$\mathrm{Na}$ imagem a seguir notamos a presença da acadêmica da Universidade Estadual do Oeste do Paraná (Unioeste), em mobilidade stricto sensu no México. No seu relato, a aluna narra desde as diferenças gastronômicas até às linguísticas, mesmo que aparentemente similares à língua portuguesa, têm suas peculiaridades e desafios a serem enfrentados para que a produção do conhecimento aconteça da melhor forma. 


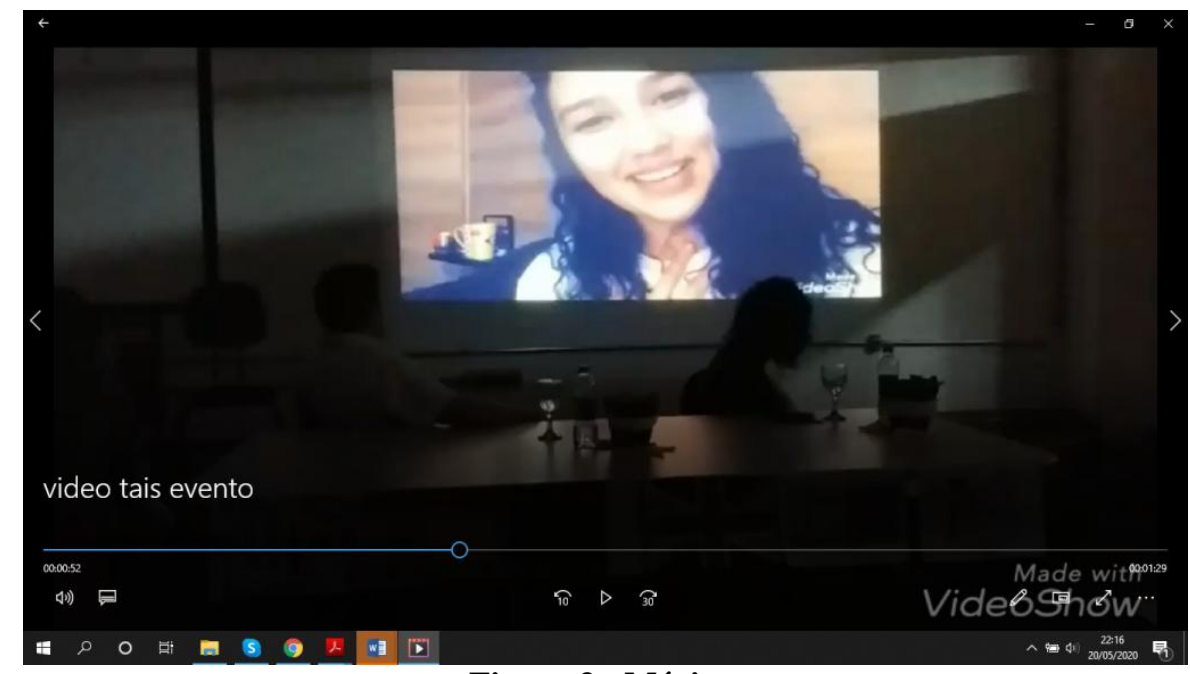

Figura 3 - México.

Fonte: https://www.youtube.com/watch?v=YxBZ5jJ9EAM\&t=6s

Além do México, também foi inserida a presença da Colômbia, no sentido do Brasil receber um acadêmico colombiano em mobilidade para fins de formação acadêmica stricto sensu.

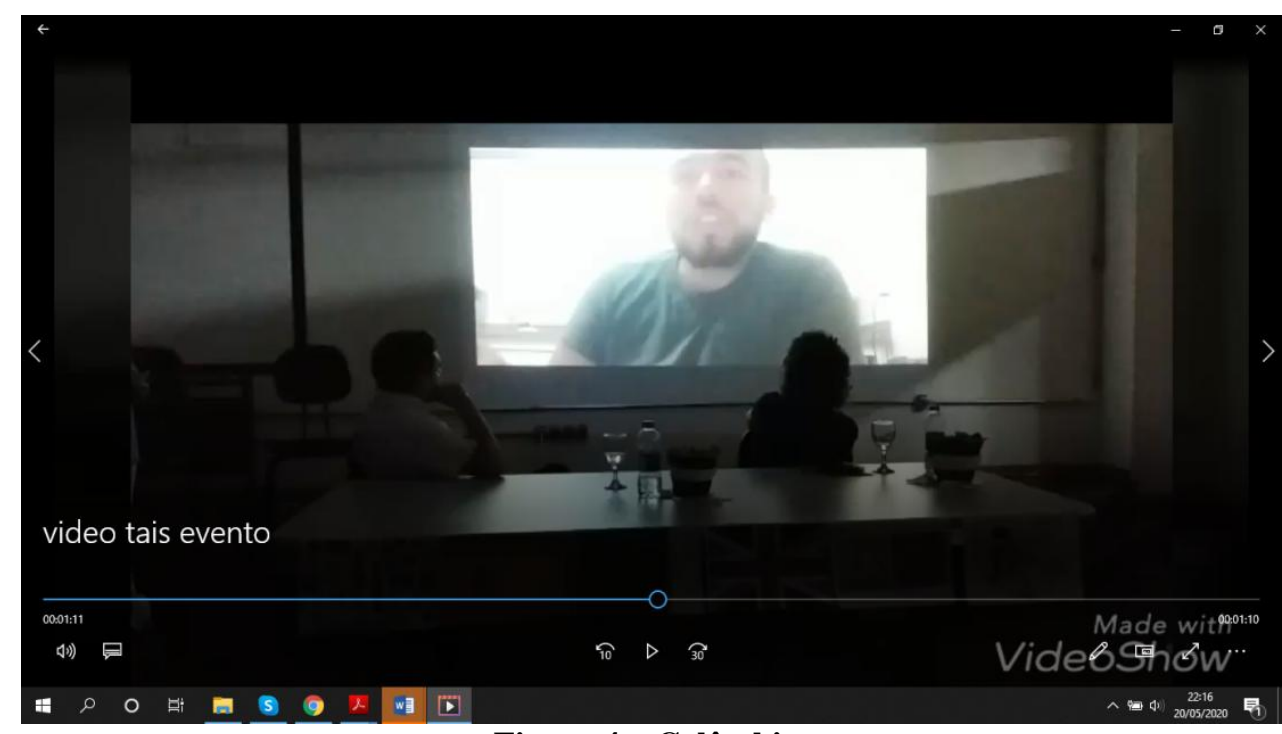

Figura 4 - Colômbia.

Fonte: https://www.youtube.com/watch?v=YxBZ5jJ9EAM\&t=6s

Sobre as contribuições latino-americanas relacionadas à produção de conhecimento, Perrota (2018, p. 32) constata que

las contribuciones latinoamericanas al campo de estudios de la integración formaron parte de un proceso colectivo de producción de conocimiento relacionado al fortalecimento de las Ciencias Sociales en la región desde una posición de autonomía académica en vistas a su usabilidad ya que se partía del supuesto de compromiso social con la resolución de problemas cognitivos locales y contextuales. 
Desse modo, verificamos o papel das Ciências Sociais no fortalecimento das relações entre os países da América Latina, o que nos ajuda a pensar que encontros como estes podem consolidar a existência de uma rede mais conectada e engajada nas questões concernentes às problemáticas não somente globais, mas pontuais à América Latina.

\section{Resultados}

Diante das discussões realizadas em torno do assunto, verificamos que os envolvidos no encontro exerceram de alguma forma uma espécie de poder, uma simbólica influência nos contatos estabelecidos nos países de origem e nos países de mobilidade, como descrito na tabela abaixo:

\begin{tabular}{|c|c|}
\hline Função & País de experiência acadêmica internacional \\
\hline Acadêmica da Unioeste & México \\
\hline Acadêmico colombiano & Brasil \\
\hline Professor da UTFPR-FB & Catar \\
\hline Egressa da UTFPR-FB & País de Gales \\
\hline Egresso da UTFPR-FB & França \\
\hline Servidora da UTFPR-FB & Portugal \\
\hline
\end{tabular}

Tabela 1 - Participantes do encontro sobre internacionalização Fonte: https://www.youtube.com/watch?v=YxBZ5jJ9EAM\&t=6s

Nesse sentido, verificamos que locais considerados geograficamente à margem das relações de MEI foram visitados e trouxeram consigo a possibilidade de difundir o soft power mencionado por Nye (2004) e Hoogwaerts (2016).

Além disso, a integração latino-americana discutidas por Perrota (2018) teve efeitos no encontro realizado a partir das experiências colombianas e mexicanas retratadas nas relações de mobilidade internacional, além de veiculação nas mídias institucionais que circularam na UTFPR através da Revista Tecnológica, por exemplo.

\section{Conclusões}

Na sociedade globalizada descrita por Bauman (1999) o mercado, a educação e as relações culturais se atravessam e formam esta necessidade mundial de internacionalizar para 
ter acesso à novas técnicas, novos conhecimentos, culturas e para alcançar melhores chances no mercado de trabalho.

A forma, no entanto, com que as IES estabelecem essas relações globalizadas podem ou não gerar efeitos de ruptura com as relações eurocêntricas e norte-americanas, as quais já estão postas.

Embora representado uma pequena manifestação de soft power, o encontro realizado pelos graduandos de Licenciatura em Informática, turma 2018.2 da UTFPR-FB quis transpor os limites do discurso secular da internacionalização, para oportunizar outras alternativas de se pensar a mobilidade acadêmica internacional pelas vias tanto das culturas desconhecidas para muitos de nós, no caso do Catar, assim como estreitar laços latino-americanos, exemplificados pela Colômbia e México.

Nesse sentido, esperamos ter contribuído, ainda que de modo incipiente, para o debate teórico e para as práticas acadêmicas.

\section{Referências}

ALFANO, B. 'A educação deve ser pensada durante a vida inteira', diz Zygmunt Bauman. Criador do conceito de 'modernidade líquida', filósofo vai palestrar no encontro Educação 360. O Globo, publicação online, 23/08/2016. Disponível em: http://oglobo.globo.com/sociedade/educacao/a-educacao-deve-ser-pensada-durante-vidainteira-diz-zygmunt-bauman-17275423\#ixzz4VsbqH7hK. Acesso em: 25 maio 2020.

BAUER, M. W; GASKEL, G. Pesquisa qualitativa com texto, imagem e som: um manual prático I tradução de Pedrinho A. Guareschi. Petrópolis, RJ: Vozes, 2002.

BAUMAN, Z. Globalização: as consequências humanas. Trad. Marcus Penchel. Rio de Janeiro: Jorge Zahar Ed., 1999.

BRASIL. Lei $\mathbf{n}^{\mathbf{0}}$ 11.184, de 7 de outubro de 2005. Disponível em: http://www.utfpr.edu.br/documentos/reitoria/documentos-institucionais/pdi. Acesso em: 25 maio 2020.

ENCONTRO ACADÊMICO. Disponível em: https://www.youtube.com/watch?v=YxBZ5jJ9EAM\&t=6s. Acesso em: 25 maio 2020.

HOOGWAERTS, L. Museums, exchanges, and their contribution to Joseph Nye's concept of 'soft power'. Museum \& Society, July. 14 (2) 313-322, 2016.

IBGE. Países. Disponível em: https://paises.ibge.gov.br/\#/. Acesso em: 25 jun. 2020.

LINGNAU, C.M.; NAVARRO, P. Ciência sem fronteiras como acontecimento discursivo e dispositivo de poder/saber. Educação em Revista (online), v. 34, p. 1-24, 2018. 
MERCOSUL EDUCACIONAL. Disponível em: http://www.edu.mercosur.int/pt-BR/ Acesso em: 24 maio 2020.

MOROSINI, M. C. Estado do conhecimento sobre internacionalização da educação superior: conceitos e práticas. Educar. Curitiba, n. 28, 2006.

NEZ, E.; MOROSINI, M. C. A cooperação acadêmica e os processos de internacionalização. Revista Panorâmica, v. 1, p. 52-65, 2020.

NYE, Jr., J. S. Soft Power: The Origins and Political Progress of a Concept. Palgrave Communications (February 21), 2017.

PERROTA, D.V. El campo de estudios de la integración regional y su aporte a las Relaciones Internacionales: una mirada desde América Latina. Madri, Espanha: Relaciones Internacionales, número 38, 2018.

UTFPR. Plano de desenvolvimento institucional (PDI) 2018-2022. Disponível em: http://www.utfpr.edu.br/documentos/reitoria/documentos-institucionais/pdi. Acesso em: 23 maio 2020.

\section{REVISTA}

TECNOLÓGICA.

Disponível

em:

http://www.utfpr.edu.br/comunicacao/produtos/revista-tecnologica/revista-tecnologicaedicao-8.pdf/view. Acesso em: 25 maio 2020. 